\begin{tabular}{|c|l|}
\hline Title & Boltzmann machine neuron circuit using single electron tunneling \\
\hline Author(s) & A kazawa, Masamichi; A memiya, Y oshihito \\
\hline Citation & $\begin{array}{l}\text { A pplied Physics Letters, 70(5), 670-672 } \\
\text { https://doi.org/10.1063/118329 }\end{array}$ \\
\hline Issue Date & 1997-02-03 \\
\hline Doc URL & http://hdl.handle.net/2115/5747 \\
\hline Rights & Copyright $\odot 1997$ A merican Institute of Physics \\
\hline Type & article \\
\hline File Information & APL 70-5.pdf \\
\hline
\end{tabular}

Instructions for use 


\title{
Boltzmann machine neuron circuit using single-electron tunneling
}

\author{
Masamichi Akazawa ${ }^{\text {a) }}$ and Yoshihito Amemiya \\ Faculty of Engineering, Hokkaido University, Kita 13, Nishi 8, Sapporo 060, Japan
}

(Received 6 May 1996; accepted for publication 26 November 1996)

\begin{abstract}
The inherent stochastic character of single-electron tunneling can be effectively utilized for creating novel electronic circuits having high-level functions. As a sample application, we present a stochastic-response circuit for implementing Boltzmann machine neurons. The circuit consists of a single-electron circuit operating under unstable conditions. It can produce an output of a random $1-0$ bit stream with the probability for an output of 1 controlled by an input signal-a task that is difficult for conventional circuits using ordinary electronic devices. (C) 1997 American Institute of Physics. [S0003-6951(97)01505-2]
\end{abstract}

One of the promising areas of research on single-carrier electronics is that of the development of functional circuits that utilize the inherent stochastic character of the singleelectron tunneling (SET) phenomenon. In this approach, it is expected that novel electronic circuits having high-level functions will be created. In this letter, we propose one such circuit, namely, a single-electron Boltzmann machine neuron circuit.

The Boltzmann machine is a kind of feedback neural network that can solve various problems in areas such as combinatorial optimization, classification, and association. It consists of a large network of many identical processing elements, called neurons, that are interconnected bidirectionally with links, or signal connections, that have various connection strengths. Each neuron receives input signals from other neurons and sends output signals to other neurons. The neuron has two output states, either 1 or 0 , and changes its state according to the inputs following a stochastic transition rule. Each neuron operates in parallel and adjusts its own state to those of all the others. After some processing time, all the neurons finally reach maximal consensus as to their individual states, and the whole network then stabilizes in a global configuration (see Refs. 1 and 2 for details).

The structure of mathematical problems such as combinatorial optimization can be mapped onto the structure of a Boltzmann machine by deciding on the connection pattern and connection strengths of the neurons. In this way, finding the optimal solution to a problem can be reduced to determining the optimal configuration of the Boltzmann machine. The unique and important feature of the Boltzmann machine is its method of operation; it uses stochastic neuron-state transition and simulated annealing algorithms. This allows the Boltzmann machine to reach a configuration that is globally optimal (and thereby an optimal solution) without falling into configurations that are only locally optimal. (This is a problem with other neural network models.) Because of this, the stochastic output of the neuron is the most important feature of the Boltzmann machine.

The basic concept of the Boltzmann machine neuron is illustrated in Fig. 1. It has two constituents, a sum-of-product unit and a stochastic-response unit. The sum-of-product unit has a number of input connections and local memory that stores connection strengths $w_{i}$ (positive or negative analog

${ }^{a)}$ Electronic mail: akazawa@sapiens.huee.hokudai.ac.jp values). It also receives input signals $x_{i}$ ( 1 or 0 ) (and bias input that controls threshold of neuron) from other neurons and produces the weighted sum of inputs $s\left(=\sum w_{i} x_{i}+w_{0}\right)$. The stochastic-response unit is peculiar to the Boltzmann machine neuron. It generates an output, 1 or 0 , updating the output state at every moment, following a given probability depending on the input value of $s$. The probability function for a state 1 is usually chosen to be the sigmoid function, expressed as

$$
f(s)=\frac{1}{1+\exp (-s / T)} \text { or } f(s)=\frac{1}{1+\exp (s / T)},
$$

where $T$ is the control parameter that slowly decreases from a large value to zero during the simulated annealing process. The shape of this function is shown in Fig. 2. The probability function need not necessarily be this function; any monotonic nonlinear function can be used, provided that it becomes 1 (or 0 ) at large positive values of $s$ and becomes 0 (or 1) at large negative values of $s$.

A Boltzmann machine large-scale integrated (LSI) device for practical use must integrate thousands of neurons on a chip. The crucial problem in developing such LSIs is how to implement the generation of randomness for the stochastic operation. Every neuron has to have its own randomness generator because stochastic independence between the neurons is required. But presently available circuits for generating randomness, such as the thermal noise amplifier and the

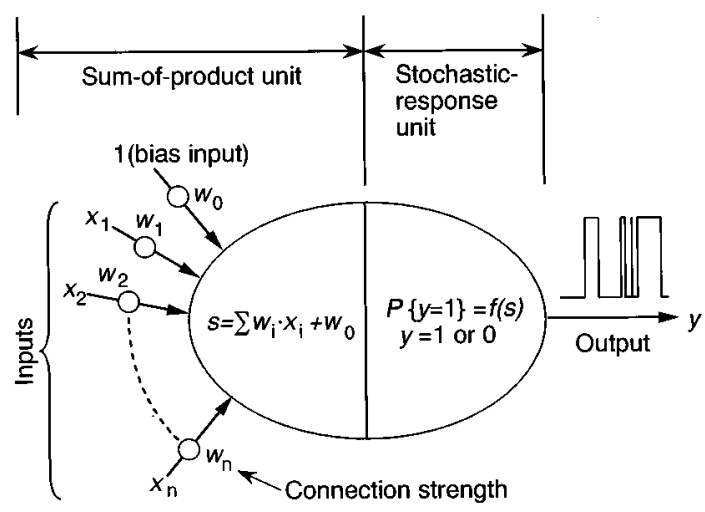

FIG. 1. Concept of a Boltzmann machine neuron. It consists of a sum-ofproduct unit and a stochastic-response unit. 


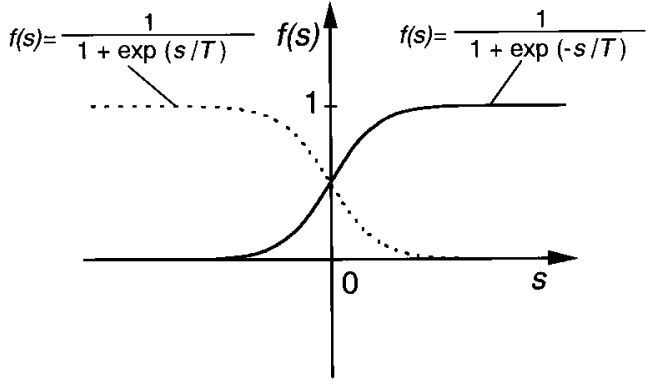

FIG. 2. Probability function $f(s)$ as a function of a weighted sum of inputs $s$. Illustrated are two functions: $f(s)=1 /[1+\exp (-s / T)]$ and $f(s)=1 /[1+\exp (s / T)]$, where $T$ is a control parameter.

random bit generator, consist of many devices and consequently require a large area; hence, they cannot be used for LSI implementation.

To overcome this problem, we present the idea that the inherent stochastic character of SET can be used for implementing the stochastic-response unit of the Boltzmann machine neuron. The objective is to operate a SET inverter under unstable conditions to produce stochastic output. Conventional inverters are designed so that they operate under bistable conditions, i.e., output 1 for low inputs and 0 for high inputs. In contrast, we propose operating a conventional SET inverter under unstable conditions in which state transition frequently occurs, leading to output of a random 1-0 bit stream. If the probability for an output of 1 (or 0 ) can be controlled by the input, this phenomenon will be useful for the stochastic-response unit of the Boltzmann machine neuron. (The circuit designed for unstable operation can be no longer called an inverter.)

Various SET-inverter circuits ${ }^{3,4}$ exist that can be used for this purpose. We here use a complementary metaloxide-semiconductor (CMOS)-type inverter circuit ${ }^{4}$ as shown in Fig. 3 and modify its stability diagram to achieve the desirable unstable condition. It consists of four tunnel junctions (the capacitances are $C_{j 1}$ and $C_{j 2}$ ), four input capacitors $\left(C_{1}, C_{2}\right)$, two bias capacitors $\left(C_{3}\right)$, and an output

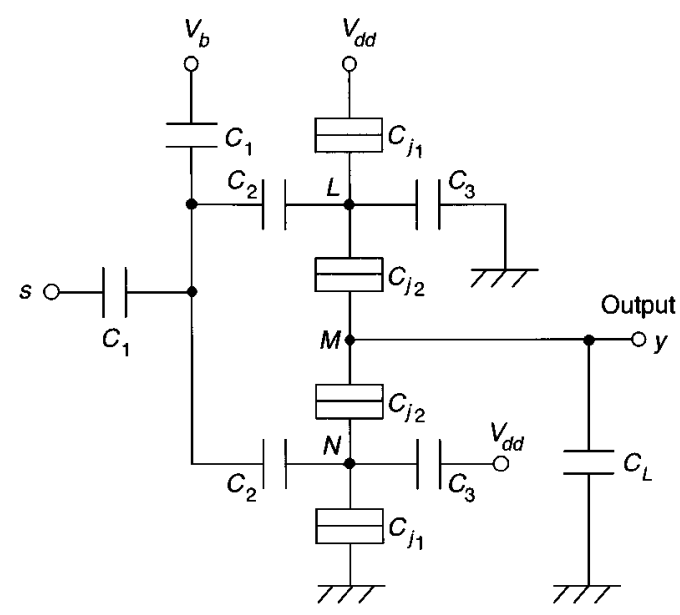

FIG. 3. An example of single-electron inverter circuits (see Ref.4). We have designed the stability diagram so that the circuit can be operated as the stochastic-response unit.

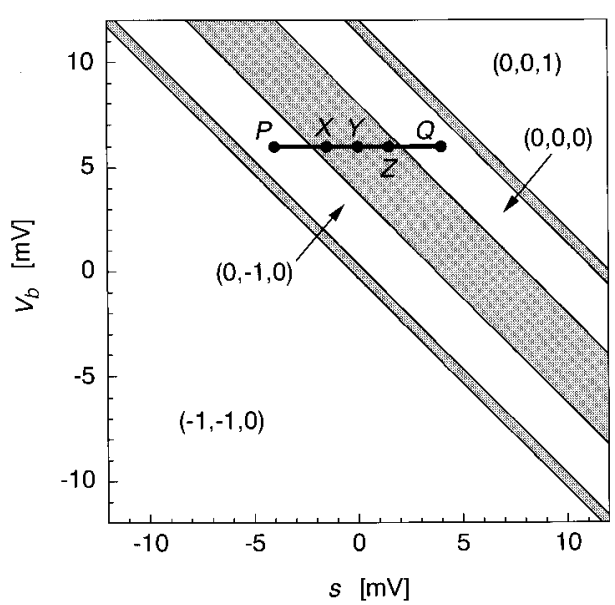

FIG. 4. A stability diagram for operating the circuit under unstable conditions. Parameters are: $C_{j 1}=1 \mathrm{aF}, C_{j 2}=2 \mathrm{aF}, C_{1}=12 \mathrm{aF}, C_{2}=4 \mathrm{aF}$, $C_{3}=10 \mathrm{aF}, C_{L}=24 \mathrm{aF}$, and $V_{d d}=6.2 \mathrm{mV}$.

capacitor $\left(C_{L}\right)$, with voltage supplies $V_{d d}$ and $V_{b}$. The circuit has three island nodes $(L, M$, and $N)$, and its internal state is expressed by the numbers of excess electrons $(l, m, n)$ stored on the three nodes respectively. The circuit receives a voltage input $s$ from a sum-of-product unit to generate its internal state and produces the corresponding voltage output $y$.

We designed the stability diagram of the circuit to operate the circuit under unstable conditions around zero input. Figure 4 illustrates a sample diagram on an $s-V_{b}$ plane, as-

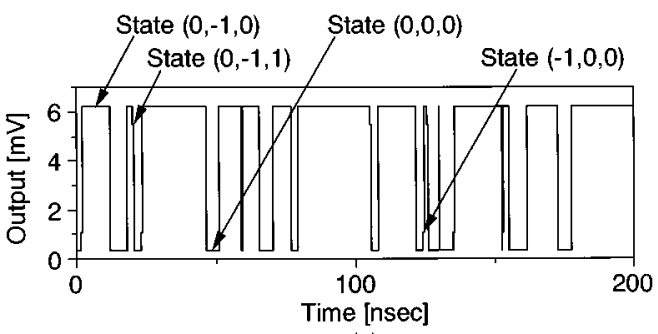

(a)

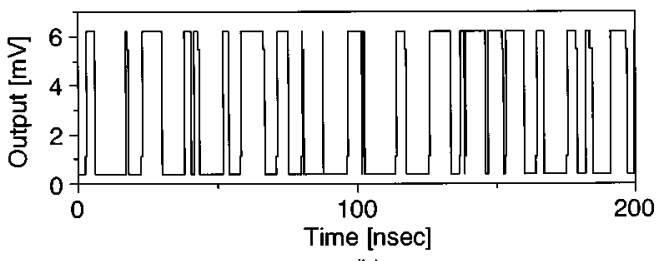

(b)

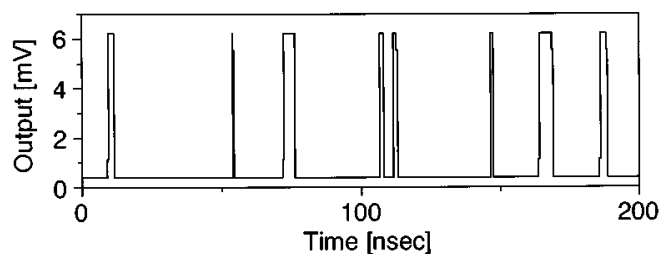

(c)

FIG. 5. Output voltage wave form for the circuit $V_{b}=6.0 \mathrm{mV}$, simulated for three input voltages, (a) $s=-1.5 \mathrm{mV}$ (point $X$ in Fig. 4), (b) $s=0 \mathrm{mV}$ (point $Y$ in Fig. 4), and (c) $s=1.5 \mathrm{mV}$ (point $Z$ in Fig. 4). The tunnel resistance is $5 \mathrm{M} \Omega$ for the four junctions and the temperature is $0 \mathrm{~K}$. 


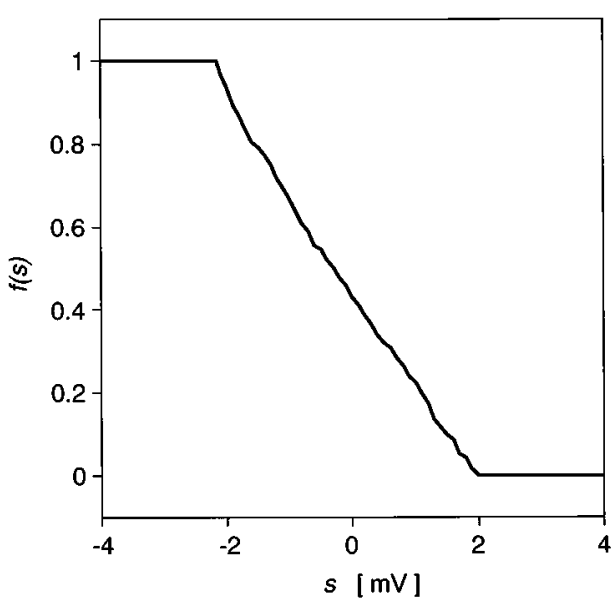

FIG. 6. Probability function for the circuit with the parameters in Fig. 4. The probability for generating an output of 1 is illustrated as a function of the input voltage $s$.

suming that $C_{j 1}=1 \mathrm{aF}, C_{j 2}=2 \mathrm{aF}, C_{1}=12 \mathrm{aF}, C_{2}=4 \mathrm{aF}$, $C_{3}=10 \mathrm{aF}, C_{L}=24 \mathrm{aF}$, and $V_{d d}=6.2 \mathrm{mV}$. Four plaincolored regions are stable zones in which the circuit stabilizes at internal state $(-1,-1,0),(0,-1,0),(0,0,0)$, and $(0$, $0,1)$; the former two states produce high positive output voltage (an output of 1), while the latter two produce low output voltage (an output of 0 ). The shaded region is an unstable zone in which electron tunneling frequently occurs and the circuit consequently alternates two or more internal states. Here we operate the circuit with $V_{b}=6.0 \mathrm{mV}$ and apply the input voltage $s$ so as to move the bias point on a segment $P Q$ from the $(0,-1,0)$ region to the $(0,0,0)$ region. It can then be expected that the probability for generation of an output of 1 depends on the input voltage $s$. The probability can be changed from 1 to 0 continuously by moving the bias point; thus $P \rightarrow X \rightarrow Y \rightarrow Z \rightarrow Q$.

We simulated circuit operation by using the Monte Carlo method $^{5}$ combined with basic equations for electric-charge distribution, charging energy, and tunneling probability. The temperature is assumed to be $0 \mathrm{~K}$. The results for the circuit in Fig. 4 are illustrated in Fig. 5. The latter figure shows the output voltage wave form (a random 1-0 bit stream) for three sample values of the input voltage under unstable conditions, i.e., $s=-1.5 \mathrm{mV}$ (point $X$ in Fig. 4), $s=0 \mathrm{mV}$ (point $Y$ in Fig. 4), and $s=1.5 \mathrm{mV}$ (point $Z$ in Fig. 4). It can be seen that the probability for an output of 1 can be controlled by the input $s$. Generally speaking, the state $(1,-1,0)$ is dominant for a low value of $s$, whereas $(0,0$, $-1)$ is dominant for a high value of $s$. Intermediate states can also be generated, but this is not a problem because their duration is always short regardless of the input voltage value. In this example, the circuit changes its internal state in a cycle of $(0,0,0) \rightarrow(-1,0,0) \rightarrow(0,-1,0) \rightarrow(0,-1,1)$ $\rightarrow(0,0,0)$.

The probability for an output of 1 is illustrated in Fig. 6 as a function of the input voltage. It is obtained by observing the output 1-0 stream for $1 \mu \mathrm{s}$ and measuring the total duration of an output of 1 . It can be seen that a probability function required for the Boltzmann machine can be obtained very easily. We will be able to fabricate Boltzmann machine LSIs compactly using single-electron circuits.

This work was supported by a Grant-in-Aid for Scientific Research on Priority Areas "Single-Electron Devices and Their High Density Integration"' (Grant No. 08247104) from the Ministry of Education, Science, Sports and Culture.

${ }^{1}$ G. E. Hinton and T. J. Sejnowski, in Parallel Distributed Processing: Explorations in the Microstructure of Cognition, edited by D. E. Rumelhart, J. L. McClelland, and the PDP Research Group (Bradford, Cambridge, 1986), Vol. 1, p. 282.

${ }^{2}$ E. Aarts and J. Korst, Simulated Annealing and Boltzmann Machines (Wiley, Chichester, 1989), p. 117

${ }^{3}$ D. V. Averin and K. K. Likharev, in Single Charge Tunneling: Coulomb Blockade Phenomena in Nanostructures, edited by H. Grabert and M. H. Devoret (Plenum, New York, 1992), p. 324.

${ }^{4}$ J. R. Tucker, J. Appl. Phys. 72, 4399 (1992).

${ }^{5}$ N. Kuwamura, K. Taniguchi, and C. Hamaguchi, Trans. IEICE J77-C-II, 221 (1994). 